\title{
Robust Optimization Approximation for Ambiguous P-Model and Its Application
}

\author{
Ru-Ru Jia ${ }^{1}$ and Xue-Jie Bai $\mathbb{D D}^{2}$ \\ ${ }^{1}$ Risk Management \& Financial Engineering Laboratory, College of Mathematics and Information Science, \\ Hebei University, Baoding, Hebei 071002, China \\ ${ }^{2}$ College of Science, Hebei Agricultural University, Baoding, Hebei 071001, China
}

Correspondence should be addressed to Xue-Jie Bai; hebaubxj@163.com

Received 15 May 2018; Accepted 28 June 2018; Published 18 July 2018

Academic Editor: A. M. Bastos Pereira

Copyright (C) $2018 \mathrm{Ru}$-Ru Jia and Xue-Jie Bai. This is an open access article distributed under the Creative Commons Attribution License, which permits unrestricted use, distribution, and reproduction in any medium, provided the original work is properly cited.

Robust optimization is a powerful and relatively novel methodology to cope with optimization problems in the presence of uncertainty. The positive aspect of robust optimization approach is its computational tractability that attracts more and more attention. In this paper, we focus on an ambiguous P-model where probability distributions are partially known. We discuss robust counterpart (RC) of uncertain linear constraints under two refined uncertain sets by robust approach and further find the safe tractable approximations of chance constraints in the ambiguous P-model. Because of the probability constraints embedded in the ambiguous P-model, it is computationally intractable. The advantage of our approach lies in choosing an implicit way to treat stochastic uncertainty models instead of solving them directly. The process above can enable the transformation of proposed P-model to a tractable deterministic one under the refined uncertainty sets. A numerical example about portfolio selection demonstrates that the ambiguous P-model can help the decision maker to determine the optimal investment proportions of various stocks. Sensitivity analyses explore the trade-off between optimization and robustness by adjusting parameter values. Comparison study is conducted to validate the benefit of our ambiguous P-model.

\section{Introduction}

Robust optimization, first proposed by Soyster [1], is a technology for dealing with optimization problems with uncertainty. This topic was widely discussed successively by Ben-Tal and Nemirovski $[2,3]$, who proved that the robust convex formulation of an uncertain problem is tractable. An additional attempt was made by Bertsimas et al. [4] and Bertsimas and Sim [5] to further develop robust optimization theory. Bertsimas et al. [4] explored the robust counterpart of a problem with an uncertainty set. Bertsimas and Sim [5] discussed the tractability for different types of robust problems. The key issue of robust optimization is to model the uncertainty. The uncertain data of a robust optimization problem vary in an uncertainty set. A vital feature of the robust optimization approach is its tractability whenever the uncertainty set itself is computationally tractable. For a thorough coverage of developments and recent advances in robust optimization, the interested reader can refer to Gabrel et al. [6].

In recent years, robust optimization has gained extensive attention in a great deal of areas. For example, Bai and Liu [7] developed an uncertain supply chain network design model to guide the decision maker with robust location-allocation strategy. Jabbarzadeh et al. [8] presented a stochastic robust optimization model for the design of a closed-loop supply chain network under uncertain weights varying in the box uncertainty set. Omrani [9] introduced a robust optimization approach to find common weights in DEA under the ellipsoidal uncertainty set. Shokouhi et al. [10] employed a robust optimization approach for imprecise data envelopment analysis under uncertain input and output parameters varying in the box uncertainty set. Bruni et al. [11] proposed an adaptive robust optimization model for the resource allocation decisions under uncertain activity durations varying in the budgeted uncertainty set. Neyshabouri and Berg [12] 
considered a two-stage robust optimization model to elective surgery and downstream capacity planning under uncertain parameters varying in the budgeted uncertainty set. Liu et al. [13] applied distributionally robust optimization approach to multiproduct newsvendor problem under uncertain probability distribution of market demand and uncertain carbon emission. Liu et al. [14] introduced a robust optimization method for relief logistics planning under uncertainties in demand and transportation time and deduced the robust counterpart of the proposed stochastic model under the budgeted uncertainty set. Zhang and Hai [15] utilized a $\mathrm{RC}$ approach to the biobjective emergency medical service design problem under the uncertain parameters varying in the ellipsoidal uncertainty set.

Robust optimization approach, as a tractable alternative to stochastic optimization, can provide a safe approximation to chance constraint under uncertainty. Ben-Tal et al. [16] discussed some approximations of chance constraints, such as robust safe approximations of ambiguous chance constraints under box, ball, box + ball, and box + budgeted uncertainty sets. $\mathrm{Li}$ and $\mathrm{Li}$ [17] studied the optimal robust optimization approximations for chance constraints under box and ellipsoidal uncertainty sets by adjusting the size of uncertainty sets. Nemirovski [18] addressed robust counterpart forms of Bernstein approximations of chance constraints under ball + box, ball, budgeted, and box uncertainty sets. Düzgün and Thiele [19] addressed a safe tractable approximation of chance constraints by robust optimization, when only the first two moments and the support of the random parameters were known. Yang and Xu [20] investigated the computational aspects of chance constraints under uncertain distribution by distributionally robust optimization. Postek et al. [21] utilized distributionally robust optimization to study ambiguous stochastic constraints under partial probability distribution consisting of means and dispersion measures of the underlying random parameters. Qiu et al. [22] considered the tractable robust formulation under the assumption of ellipsoid discrete distribution and again under box discrete distribution by distributionally robust optimization. Zymler et al. [23] studied the tractable approximations of joint chance constraints under second-order moment information by robust distributional optimization.

The above researches applied robust optimization method to different fields under box, budgeted, and ellipsoidal uncertainty sets and built safe approximations of chance constraints under box, budget, box + budget, box + ball, and ellipsoidal uncertainty sets, none of which are based on box + ellipsoidal set or box + generalized budgeted set. In this paper, we focus on an ambiguous P-model under box + generalized budgeted and box + ellipsoidal uncertainty sets. Then, we utilize robust optimization approach to build the safe tractable approximations of the chance constraints for our ambiguous P-model under box + generalized budgeted and box + ellipsoidal uncertainty sets, respectively, to resist computationally intractable problem of chance constraints.

To illustrate the efficiency of the ambiguous probability model, we present a numerical example based on portfolio selection problem. Portfolio optimization is to allocate limited capital on the available financial assets to achieve a trade-off between risk and return. There are a lot of studies on portfolio selection problems. For example, Bai and Liu [24] introduced the second-order moment of fuzzy variables and constructed a class of mean-moment fuzzy portfolio optimization models. Liu et al. [25] studied portfolio selection problem under credibilistic CVaR criterion. Bruni et al. [26] proposed the exact and approximate stochastic dominance strategies for portfolio selection. Ling and $\mathrm{Xu}$ [27] explored robust portfolio selection models under a "marginal + joint" ellipsoidal uncertainty set. Gülpinar and Canakoḡlu [28] considered a portfolio selection problem by robust optimization approach under temperature uncertainty using scenariobased as well as symmetric ellipsoidal and asymmetric uncertainty sets. Gregory et al. [29] evaluated the cost of robustness for the robust counterpart to the maximum return portfolio optimization problem under uncertain returns varying in the ellipsoidal uncertainty sets. Unlike these documents, we utilize robust optimization approach to model a portfolio selection problem under the uncertain return rates varying in the box + ellipsoidal and box + generalized budget uncertainty sets.

The main contributions of this paper include the following aspects. Firstly, we focus on an ambiguous P-model and deduce its robust counterpart under two refined uncertainty sets: box + generalized budgeted set and box + ellipsoidal set that are less conservative than unrefined uncertainty sets. Secondly, we utilize robust optimization approach to build the safe tractable approximations of the ambiguous Pmodel in the case of box + generalized budgeted and box + ellipsoidal uncertainty sets, respectively. This transformation reduces greatly computational complexity and makes the application of our procedure more understandable. Thirdly, the effectiveness of proposed ambiguous P-model is demonstrated through a portfolio selection problem.

The remainder of this paper is organized as follows. In Section 2 we introduce a kind of ambiguous P-model. Section 3 describes the safe approximations of chance constraints for the ambiguous P-model under different uncertainty sets. In Section 4 we present a numerical example about portfolio selection problem to illustrate the efficiency of the proposed model. Section 5 concludes the paper.

\section{General Formulation of Ambiguous P-Model}

The first formulation of P-model was presented by Charnes and Cooper [30]. Charnes and Cooper [31] and Charnes and Kirby [32] further discussed some special P-models in chance-constrained programming. There are some applications of P-model like communications, weather forecasts, and mass production under an exact specification of the probability distribution.

In real-world problems, the exact probability distribution information of uncertain parameter is often unavailable. Many researchers considered the imprecise probability in the existing literature. For example, Walley [33] stated the general imprecise probability as well as its application in statistical reasoning. Walley et al. [34] provided general algorithms 
for making inferences from any finite collection of (possibly imprecise) conditional probabilities. Cozman and Walley [35] considered the existence of imprecise probability. Different from works discussed above, we focus on an ambiguous Pmodel where the true distribution is only partially known and belongs to a given family of distributions. The general formulation of the ambiguous P-model is the following:

$$
\begin{array}{ll}
\min & \epsilon \\
\text { s.t. } \quad \operatorname{Pr}_{(c, d) \sim p}\left\{c^{T} x+d \leq \bar{t}\right\} \geq 1-\epsilon, \quad \forall p \in \wp \\
\\
\operatorname{Pr}_{\left(a_{i}, b_{i}\right) \sim p_{i}}\left\{a_{i}^{T} x \leq b_{i}\right\} \geq 1-\epsilon_{i}, \\
\forall i=1,2, \ldots, m, p_{i} \in \wp_{i} .
\end{array}
$$

In model (1), $x \in \mathbb{R}^{n}$ is the vector of decision variables, and $c, a_{i} \in \mathbb{R}^{n}$ and $d, b_{i} \in \mathbb{R}$ form the chance constraints. The objective function is to minimize the probability level $\epsilon \in(0,1)$. Parameter $\epsilon_{i} \in(0,1)$ is a prespecified small tolerance and $\bar{t}$ is a known constant. $\operatorname{Pr}_{(c, d) \sim p}$ and $\operatorname{Pr}_{\left(a_{i}, b_{i}\right) \sim p}$ are the probabilities associated with the distributions $p$ and $p_{i}$ that belong to the given families $\wp$ and $\wp_{i}$ of distributions, respectively. The possible values of the data $(c, d),\left(a_{i}, b_{i}\right)$ are varying in given uncertainty sets. The uncertainty sets are parameterized, in an affine way, by perturbation vectors $\zeta=$ $\left(\zeta_{1}, \zeta_{2}, \ldots, \zeta_{L}\right)$ and $\zeta_{i}=\left(\zeta_{1}, \zeta_{2}, \ldots, \zeta_{H_{i}}\right)$ varying in given perturbation sets $Z$ and $Z_{i}$, respectively. Therefore, the bodies of the chance constraints within model (1) can be written as

$$
\begin{aligned}
& c^{T} x+d \leq \bar{t}, \\
& \mathcal{U}=\left\{[c ; d]=\left[c^{0} ; d^{0}\right]+\sum_{l=1}^{L} \zeta_{l}\left[c^{l} ; d^{l}\right]: \forall \zeta \in Z\right\}, \\
& a_{i}^{T} x \leq b_{i}, \\
& \mathscr{U}_{i}=\left\{\left[a_{i} ; b_{i}\right]=\left[a_{i}^{0} ; b_{i}^{0}\right]+\sum_{h=1}^{H_{i}} \zeta_{i}^{h}\left[a_{i}^{h} ; b_{i}^{h}\right]: \forall \zeta_{i} \in Z_{i}\right\}, \\
& \forall i=1,2, \ldots, m,
\end{aligned}
$$

where $\left[c ; d ; a_{i} ; b_{i}\right]$ represent basic perturbations from the nominal data $\left[c^{0} ; d^{0} ; a_{i}^{0} ; b_{i}^{0}\right]$.

We reformulate model (1) as follows:

$$
\begin{aligned}
& \min \epsilon \\
& \text { s.t. } \quad \operatorname{Pr}_{\zeta \sim p}\left\{\zeta:\left[c^{0}\right]^{T} x+\sum_{l=1}^{L} \zeta_{l}\left[c^{l}\right]^{T} x+d^{0}+\sum_{l=1}^{L} \zeta_{l} d^{l}>\bar{t}\right\} \leq \epsilon, \\
& \forall p \in \wp, \zeta \in Z \\
& \\
& \operatorname{Pr}_{\zeta_{i} \sim p_{i}}\left\{\zeta_{i}:\left[a_{i}^{0}\right]^{T} x+\sum_{h=1}^{H_{i}} \zeta_{i}^{h}\left[a_{i}^{h}\right]^{T} x>b_{i}^{0}+\sum_{h=1}^{H_{i}} \zeta_{i}^{h} b_{i}^{h}\right\} \\
& \leq \epsilon_{i}, \quad \forall p_{i} \in \wp_{i}, \zeta_{i} \in Z_{i}, i=1,2, \ldots, m .
\end{aligned}
$$

The ambiguous P-model is a significant tool for modeling the real-world optimization problems with uncertainty. However, the model faces the challenge of computing the optimal solution when the probability distributions are partially known. In order to build computationally tractable reformulation of the model, it is necessary to deal with the following chance constraints:

$$
\begin{aligned}
& \operatorname{Pr}_{\zeta \sim p}\left\{\zeta:\left[c^{0}\right]^{T} x+\sum_{l=1}^{L} \zeta_{l}\left[c^{l}\right]^{T} x+d^{0}+\sum_{l=1}^{L} \zeta_{l} d^{l}>\bar{t}\right\} \\
& \quad \leq \epsilon, \quad \forall p \in \wp, \zeta \in Z, \\
& \operatorname{Pr}_{\zeta_{i} \sim p_{i}}\left\{\zeta_{i}:\left[a_{i}^{0}\right]^{T} x+\sum_{h=1}^{H_{i}} \zeta_{i}^{h}\left[a_{i}^{h}\right]^{T} x>b_{i}^{0}+\sum_{h=1}^{H_{i}} \zeta_{i}^{h} b_{i}^{h}\right\} \\
& \leq \epsilon_{i}, \quad \forall p_{i} \in \wp_{i}, \quad \zeta_{i} \in Z_{i} .
\end{aligned}
$$

There are several challenging aspects of solving chance constraints [16]. (i) Due to the ambiguous probability distributions, it is difficult to formulate an equivalent deterministic constraint for chance constraints. (ii) It is not easy to check the feasibility of chance constraints. (iii) The feasible region of chance constraints is often nonconvex. Generally, there are some types of approximation techniques of chance constraints used in the literature: sampling based approach [36] and analytical approximation approach [37]. This paper chooses an implicit way to treat stochastic uncertainty models by replacing the chance constraints with their computationally tractable safe approximations. In [16], the safe approximation was defined as follows.

Definition 1 (see [16]). Let $\left[c^{l} ; d^{l}\right]_{l=0}^{L}, P, \epsilon$ be the data of chance constraints (5), and let $S$ be a system of convex constraints on $x$ and additional variables $v$. One says that $S$ is a safe convex approximation of chance constraints (5), if the $x$ component of every feasible solution $(x, v)$ of $S$ is feasible for the chance constraint. If the convex constraints forming $S$ are efficiently computable, then the safe approximating program is called computationally tractable.

\section{Safe Approximations of Chance Constraints}

In this section, we utilize a robust optimization method to extend the proposed ambiguous P-model into a robust formulation under box + generalized budgeted and box + ellipsoidal perturbation sets.

Now we employ robust optimization method to construct the safe approximations of chance constraints (5) and (6) under box + generalized budgeted and box + ellipsoidal perturbation sets, respectively. In Section 3.1 we give a detailed approximation process of constraint (5). Here we assume that all distributions satisfy the following properties, which was also considered by Nemirovski and Shapiro [37]:

$(P 1)$. Random variables $\zeta_{l}, l=1, \ldots, L$, are independent.

$(P 2)$. The distributions $P_{l}$ of the components $\zeta_{l}$ are such that

$$
\int \exp \{t s\} \mathrm{d} P_{l}(s) \leq \exp \left\{\max \left[\mu_{l}^{+} t, \mu_{l}^{-} t\right]+\frac{1}{2} \sigma_{l}^{2} t^{2}\right\},
$$

$\forall t \in \mathbb{R}$,

with known constants $\mu_{l}^{+} \geq \mu_{l}^{-}$and $\sigma_{l} \geq 0$. 
Without loss of generality, we assume $\mu_{l}^{+}=\mu_{l}^{-}=0$ in this paper.

3.1. The Safe Approximation under Box + Generalized Budgeted Perturbation Set. In the subsection, we are about to find the safe approximations of chance constraints (5) and (6) with box + generalized budgeted perturbation set.

First of all, we set out to find the RC of (2) and (3) under box + generalized budgeted perturbation set.

Set $Z$ as the intersection of the box and generalized budget centered at the origin:

$$
\begin{aligned}
& Z \\
& =\left\{\zeta \in \mathbb{R}^{L}:-1 \leq \zeta_{l} \leq 1, \sum_{l=1}^{L}\left|\frac{\zeta_{l}}{\sigma_{l}}\right| \leq \gamma, l=1, \ldots, L\right\},
\end{aligned}
$$

where $\sigma_{l}>0$ and $1 \leq \gamma \leq L$ are parameters.

Here the conic representation becomes

$$
Z=\left\{\zeta \in \mathbb{R}^{L}: Q_{1} \zeta+q_{1} \in \mathbf{K}^{1}, Q_{2} \zeta+q_{2} \in \mathbf{K}^{2}\right\}
$$

where

(i) $Q_{1} \zeta \equiv[\zeta ; 0], q_{1}=\left[0_{L \times 1} ; 1\right]$, and $\mathbf{K}^{1}=\left\{(y, t) \in \mathbb{R}^{L} \times\right.$ $\left.\mathbb{R}: t \geq\|y\|_{\infty}\right\}$, whence $\mathbf{K}_{*}^{1}=\left\{(y, t) \in \mathbb{R}^{L} \times \mathbb{R}: t \geq\right.$ $\left.\|y\|_{1}\right\}$, which is referred to as the cone dual to $\mathbf{K}^{1}$;

(ii) $Q_{2} \zeta=\left[\Sigma^{-1} \zeta ; 0\right]$ with $\sum=\operatorname{Diag}\left\{\sigma_{1}, \ldots, \sigma_{L}\right\}$ that represents the diagonal matrix, $q_{2}=\left[0_{L \times 1} ; \gamma\right]$ and $\mathbf{K}^{2}=$ $\mathbf{K}_{*}^{1}$, whence $\mathbf{K}_{*}^{2}=\mathbf{K}^{1}$.

Setting $\varphi^{1}=\left[\kappa_{1} ; \rho_{1}\right] \in \mathbf{K}_{*}^{1}, \varphi^{2}=\left[\kappa_{2} ; \rho_{2}\right] \in \mathbf{K}_{*}^{2}$ with onedimensional $\rho_{1}, \rho_{2}$ and $L$-dimensional $\kappa_{1}, \kappa_{2}$, inequality (2) becomes the following system of constraints in variables $\rho_{1}$, $\rho_{2}, \kappa_{1}, \kappa_{2}, x$ :

$$
\begin{aligned}
q_{1} \rho_{1}+q_{2} \rho_{2}+\left[c^{0}\right]^{T} x+d^{0} \leq \bar{t}, & \\
\left(Q_{1} \kappa_{1}+Q_{2} \kappa_{2}\right)_{l}=-d^{l}-\left[c^{l}\right]^{T} x, & l=1, \ldots, L, \\
\left\|\kappa_{1}\right\|_{1} \leq \rho_{1} & {\left[\Longleftrightarrow\left[\kappa_{1} ; \rho_{1}\right] \in \mathbf{K}_{*}^{1}\right], } \\
\left\|\kappa_{2}\right\|_{\infty} \leq \rho_{2} & {\left[\Longleftrightarrow\left[\kappa_{2} ; \rho_{2}\right] \in \mathbf{K}_{*}^{2}\right] . }
\end{aligned}
$$

These are equivalent to the following formulations:

$$
\begin{aligned}
& \rho_{1}+\gamma \rho_{2}+\left[c^{0}\right]^{T} x+d^{0} \leq \bar{t}, \\
&\left(\kappa_{1}+\Sigma^{-1} \kappa_{2}\right)_{l}=-d^{l}-\left[c^{l}\right]^{T} x, \quad l=1, \ldots, L, \\
&\left\|\kappa_{1}\right\|_{1} \leq \rho_{1} \quad\left[\Longleftrightarrow\left[\kappa_{1} ; \rho_{1}\right] \in \mathbf{K}_{*}^{1}\right], \\
&\left\|\kappa_{2}\right\|_{\infty} \leq \rho_{2} \quad\left[\Longleftrightarrow\left[\kappa_{2} ; \rho_{2}\right] \in \mathbf{K}_{*}^{2}\right] .
\end{aligned}
$$

For every feasible solution to the system, we have $\rho_{1} \geq \bar{\rho}_{1} \equiv$ $\left\|\kappa_{1}\right\|_{1}, \rho_{2} \geq \bar{\rho}_{2} \equiv\left\|\kappa_{2}\right\|_{\infty}$. The solution obtained is still feasible when replacing $\rho_{1}, \rho_{2}$ with $\bar{\rho}_{1}, \bar{\rho}_{2}$. Based on the above reasons, we can eliminate the variables $\rho_{1}, \rho_{2}$ from this system. The reduced system in variables $x, \kappa_{1}, \kappa_{2}$ reads

$$
\begin{aligned}
&\left\|\kappa_{1}\right\|_{1}+\gamma\left\|\kappa_{2}\right\|_{\infty}+\left[c^{0}\right]^{T} x+d^{0} \leq \bar{t}, \\
&\left(\kappa_{1}+\Sigma^{-1} \kappa_{2}\right)_{l}=-d^{l}-\left[c^{l}\right]^{T} x, \\
& \quad l=1, \ldots, L .
\end{aligned}
$$

Since $p-$ norms $\left\|\kappa_{i}\right\|_{p}=\sum_{l=1}^{L}\left(\left\|\left(\kappa_{i}\right)_{l}\right\|^{p}\right)^{1 / p}(1 \leq p<\infty)$ and $\left\|\kappa_{i}\right\|_{\infty}=\max _{l}\left\|\left(\kappa_{i}\right)_{l}\right\|$, the simplified formulations are given by

$$
\begin{aligned}
& \sum_{l=1}^{L}\left|\left(\kappa_{1}\right)_{l}\right|+\gamma \max _{l}\left|\left(\kappa_{2}\right)_{l}\right|+\left[c^{0}\right]^{T} x+d^{0} \leq \bar{t}, \\
& \left(\kappa_{l}+\Sigma^{-1} \kappa_{2}\right)_{l}=-d^{l}-\left[c^{l}\right]^{T} x, \quad l=1, \ldots, L .
\end{aligned}
$$

For the sake of presentation, we obtain the following formulation by $r=\kappa_{l}, \eta=\Sigma^{-1} \kappa_{2}$ :

$$
\begin{aligned}
& \sum_{l=1}^{L}\left|r_{l}\right|+\gamma \max _{l}\left|\sigma_{l} \eta_{l}\right|+\left[c^{0}\right]^{T} x+d^{0} \leq \bar{t}, \\
& r_{l}+\eta_{l}=-d^{l}-\left[c^{l}\right]^{T} x, \\
& l=1, \ldots, L .
\end{aligned}
$$

The above process proves that ((14a) and (14b)) is the RC of (2).

Similarly, we can write the RC of (3):

$$
\begin{aligned}
& \sum_{h=1}^{H_{i}}\left|z_{i}^{h}\right|+\gamma_{i} \max _{h}\left|\sigma_{i}^{h} \omega_{i}^{h}\right|+\left[a_{i}^{0}\right]^{T} x \leq b_{i}^{0}, \\
& z_{i}^{h}+w_{i}^{h}=b_{i}^{h}-\left[a_{i}^{h}\right]^{T} x, \\
& h=1, \ldots, H_{i} .
\end{aligned}
$$

The RC of (2) and (3) are the explicit convex constraints.

Next, we will prove that the RC of (2) and (3) are the safe convex approximations of the chance constraints (5) and (6), respectively.

Theorem 2. Let the random perturbations affecting (2) obey $(P 1)$ and $(P 2)$, and consider the RC of (2) corresponding to the perturbation set (8). This $R C$ can be equivalently represented by the system of constraints ((14a) and (14b)). And every feasible solution to this system ((14a) and (14b)) is feasible for the chance constraint (5).

(11) Proof. The fact that ((14a) and (14b)) represents the RC of constraint (2) has been proved, with the perturbation set being (8). Now let us prove that $((14 a)$ and $(14 b))$ is the safe approximation of constraint (5). That is, if $(P 1)$ and $(P 2)$ take 
place and $x, r, \eta$ are feasible for $((14 \mathrm{a})$ and (14b)), then $x$ is feasible for constraint (5). Indeed, when $\|\zeta\|_{\infty} \leq 1$, we have

$$
\left[c^{0}\right]^{T} x+d^{0}+\sum_{l=1}^{L} \zeta_{l}\left[\left[c^{l}\right]^{T} x+d^{l}\right]>\bar{t}
$$

by (14b), the inequality can be expressed as

$$
-\sum_{l=1}^{L} r_{l} \zeta_{l}-\sum_{l=1}^{L} \eta_{l} \zeta_{l}+\left[c^{0}\right]^{T} x+d^{0}>\bar{t}
$$

Since $\|\zeta\|_{\infty} \leq 1$, we have

$$
\sum_{l=1}^{L}\left|r_{l}\right|-\sum_{l=1}^{L} \eta_{l} \zeta_{l}+\left[c^{0}\right]^{T} x+d^{0}>\bar{t}
$$

and then, by (14a), we have the following computational result:

$$
-\sum_{l=1}^{L} \eta_{l} \zeta_{l}>\gamma \max \left|\sigma_{l} \eta_{l}\right|
$$

and since $\left\|\sigma_{l} \eta_{l}\right\|_{2} \leq \sqrt{L}\left\|\sigma_{l} \eta_{l}\right\|_{\infty}$, we have

$$
-\sum_{l=1}^{L} \eta_{l} \zeta_{l}>\frac{\gamma}{\sqrt{L}} \sqrt{\sum_{l=1}^{L} \sigma_{l}^{2} \eta_{l}^{2}}
$$

Therefore, for every probability distribution compatible with $(P 2)$, we have

$$
\begin{aligned}
& \operatorname{Pr}_{\zeta \sim p}\{\zeta: x \text { is infeasible for }(2)\} \\
& \quad \leq \operatorname{Pr}_{\zeta \sim p}\left\{-\sum_{l=1}^{L} \eta_{l} \zeta_{l}>\frac{\gamma}{\sqrt{L}} \sqrt{\sum_{l=1}^{L} \sigma_{l}^{2} \eta_{l}^{2}}\right\} \\
& \quad \leq \exp \left\{-\frac{\gamma^{2}}{2 L}\right\}
\end{aligned}
$$

where the last inequality is due to

$$
\operatorname{Pr}\left\{\sum_{l=1}^{L} k_{l} \zeta_{l}>\Omega \sqrt{\sum_{l=1}^{L} \sigma_{l}^{2} k_{l}^{2}}\right\} \leq \exp \left\{-\frac{\Omega^{2}}{2}\right\}
$$

shown in [16], and parameter $\Omega$ takes its value $\sqrt{2 \ln (1 / \epsilon)}$. Thus, the quantity $\gamma / \sqrt{L}$ in our present situation plays the same role that the quantity $\Omega$ plays in this situation.

The proof of theorem is complete.

According to Theorem 2, we can conclude

$$
\begin{aligned}
& \operatorname{Pr}_{\zeta_{i} \sim p_{i}}\left\{\zeta_{i}: x \text { is infeasible for }(3)\right\} \\
& \quad \leq \operatorname{Pr}_{\zeta_{i} \sim p_{i}}\left\{-\sum_{h=1}^{H_{i}} \omega_{i}^{h} \zeta_{i}^{h}>\frac{\gamma_{i}}{\sqrt{H_{i}}} \sqrt{\sum_{h=1}^{H_{i}}\left(\sigma_{i}^{h}\right)^{2}\left(\omega_{i}^{h}\right)^{2}}\right\} \\
& \quad \leq \exp \left\{-\frac{\gamma_{i}^{2}}{2 H_{i}}\right\} .
\end{aligned}
$$

This implies that constraint $((15 a)$ and (15b)) is a safe approximation of the chance constraint (6).
Based on the analysis above, we transform our general model (4) into a RC approximation model:

$$
\begin{array}{ll}
\min & \epsilon \\
\text { s.t. } & \sum_{l=1}^{L}\left|r_{l}\right|+\sqrt{2 L \ln \left(\frac{1}{\epsilon}\right)} \max _{l}\left|\sigma_{l} \eta_{l}\right|+\left[c^{0}\right]^{T} x+d^{0} \\
& \leq \bar{t} \\
& r_{l}+\eta_{l}=-d^{l}-\left[c^{l}\right]^{T} x, \quad l=1, \ldots, L \\
& \sum_{h=1}^{H_{i}}\left|z_{i}^{h}\right|+\sqrt{2 H_{i} \ln \left(\frac{1}{\epsilon_{i}}\right)} \max _{h}\left|\sigma_{i}^{h} \omega_{i}^{h}\right|+\left[a_{i}^{0}\right]^{T} x \\
& \leq b_{i}^{0}, \quad i=1, \ldots, m \\
& z_{i}^{h}+w_{i}^{h}=b_{i}^{h}-\left[a_{i}^{h}\right]^{T} x, \\
h=1, \ldots, H_{i}, i=1, \ldots, m .
\end{array}
$$

The approximation model is the convex nonlinear programming, where every convex constraint is efficiently computable. Therefore, the above approximating programming is computationally tractable. The $x$ component of every feasible solution to the approximation is feasible for the ambiguous P-model.

3.2. The Safe Approximation under Box + Ellipsoidal Perturbation Set. In this subsection, we are about to find the safe approximations of constraints (5) and (6) with box + ellipsoidal perturbation set.

Set $Z$ is the intersection of the box and ellipsoid centered at the origin:

$$
Z=\left\{\zeta \in \mathbb{R}^{L}:-1 \leq \zeta_{l} \leq 1, \sqrt{\sum_{l=1}^{L}\left(\frac{\zeta_{l}}{\sigma_{l}}\right)^{2}} \leq \Omega, l\right.
$$

$$
=1, \ldots, L\}
$$

where $\sigma_{l}>0$ is given parameter.

Similar to the method of first cast, we can respectively write the RC of (2) and (3) as follows:

$$
\begin{aligned}
\sum_{l=1}^{L}\left|r_{l}\right|+\Omega \sqrt{\sum_{l=1}^{L} \sigma_{l}^{2} \eta_{l}^{2}+\left[c^{0}\right]^{T} x+d^{0} \leq \bar{t}} & \\
r_{l}+\eta_{l} & =-d^{l}-\left[c^{l}\right]^{T} x, \\
& l=1, \ldots, L,
\end{aligned}
$$

and

$$
\begin{aligned}
& \sum_{h=1}^{H_{i}}\left|z_{i}^{h}\right|+\Omega_{i} \sqrt{\sum_{h=1}^{H_{i}}\left(\sigma_{i}^{h}\right)^{2}\left(\omega_{i}^{h}\right)^{2}}+\left[a_{i}^{0}\right]^{T} x \leq b_{i}^{0} \\
& z_{i}^{h}+w_{i}^{h}=b_{i}^{h}-\left[a_{i}^{h}\right]^{T} x, \quad h=1, \ldots, H_{i} .
\end{aligned}
$$

We can summarize our finding in the following. 
Theorem 3. Let the random perturbations affecting (2) obey $(P 1)$ and $(P 2)$, and consider the RC of (2) corresponding to the perturbation set (25). This $R C$ can be equivalently represented by the system of conic quadratic constraints ((26a) and (26b)). And every feasible solution to this system is feasible for the chance constraint (5).

Proof. The fact is that $((26 \mathrm{a})$ and $(26 \mathrm{~b}))$ represents the $\mathrm{RC}$ of constraint (2), with the perturbation set being (25). Now let us prove that $((26 \mathrm{a})$ and $(26 \mathrm{~b}))$ is the safe approximation of constraint (5). That is, if $(P 1)$ and $(P 2)$ take place and $x$, $r, \eta$ are feasible for $((26 \mathrm{a})$ and $(26 \mathrm{~b}))$, then $x$ is feasible for constraint (5). Indeed, when $\|\zeta\|_{\infty} \leq 1$, we have

$$
\left[c^{0}\right]^{T} x+d^{0}+\sum_{l=1}^{L} \zeta_{l}\left[\left[c^{l}\right]^{T} x+d^{l}\right]>\bar{t}
$$

and, by (26b), the inequality can be expressed as

$$
-\sum_{l=1}^{L} r_{l} \zeta_{l}-\sum_{l=1}^{L} \eta_{l} \zeta_{l}+\left[c^{0}\right]^{T} x+d^{0}>\bar{t}
$$

Since $\|\zeta\|_{\infty} \leq 1$, we have

$$
\sum_{l=1}^{L}\left|r_{l}\right|-\sum_{l=1}^{L} \eta_{l} \zeta_{l}+\left[c^{0}\right]^{T} x+d^{0}>\bar{t}
$$

and then, by (26a), we have the following computational result:

$$
-\sum_{l=1}^{L} \eta_{l} \zeta_{l}>\Omega \sqrt{\sum_{l=1}^{L} \sigma_{l}^{2} \eta_{l}^{2}}
$$

Therefore, for every probability distribution compatible with $(P 2)$, we have

$$
\begin{aligned}
& \operatorname{Pr}_{\zeta \sim p}\{\zeta: x \text { is infeasible for }(2)\} \\
& \quad \leq \operatorname{Pr}_{\zeta \sim p}\left\{-\sum_{l=1}^{L} \eta_{l} \zeta_{l}>\Omega \sqrt{\sum_{l=1}^{L} \sigma_{l}^{2} \omega_{l}^{2}}\right\} \\
& \quad \leq \exp \left\{-\frac{\Omega^{2}}{2}\right\} .
\end{aligned}
$$

The proof of theorem is complete.

Based on Theorem 3, we have

$$
\begin{aligned}
& \operatorname{Pr}_{\zeta_{i} \sim p_{i}}\left\{\zeta_{i}: x \text { is infeasible for }(3)\right\} \\
& \quad \leq \operatorname{Pr}_{\zeta_{i} \sim p_{i}}\left\{-\sum_{h=1}^{H_{i}} \omega_{i}^{h} \zeta_{i}^{h}>\Omega_{i} \sqrt{\sum_{h=1}^{H_{i}}\left(\sigma_{i}^{h}\right)^{2}\left(\omega_{i}^{h}\right)^{2}}\right\} \\
& \quad \leq \exp \left\{-\frac{\Omega_{i}^{2}}{2}\right\}
\end{aligned}
$$

That is to say, constraint $((27 a)$ and $(27 b))$ is a safe approximation of chance constraint (6).
Thus, model (4) can be formulated as a RC approximation model:

$$
\begin{array}{ll}
\min & \epsilon \\
\text { s.t. } & \sum_{l=1}^{L}\left|r_{l}\right|+\sqrt{2 \ln \left(\frac{1}{\epsilon}\right)} \sqrt{\sum_{l=1}^{L} \sigma_{l}^{2} \eta_{l}^{2}}+\left[c^{0}\right]^{T} x+d^{0} \\
& \leq \bar{t} \\
& r_{l}+\eta_{l}=-d^{l}-\left[c^{l}\right]^{T} x, \quad l=1, \ldots, L \\
& \sum_{i}\left|z_{i}^{h}\right|+\sqrt{2 \ln \left(\frac{1}{\epsilon_{i}}\right)} \sqrt{\sum_{h=1}^{H_{i}}\left(\sigma_{i}^{h}\right)^{2}\left(\omega_{i}^{h}\right)^{2}} \\
& +\left[a_{i}^{0}\right]^{T} x \leq b_{i}^{0}, \quad i=1, \ldots, m \\
& z_{i}^{h}+w_{i}^{h}=b_{i}^{h}-\left[a_{i}^{h}\right]^{T} x, \\
h=1, \ldots, H_{i}, i=1, \ldots, m,
\end{array}
$$

which is computationally tractable.

\section{Robust Portfolio Selection Problem}

In the section, we present a numerical example to illustrate the efficiency of the ambiguous P-model. All mathematical models are solved by LINGO 11.0 on personal computer (Intel ${ }^{\circledR}$ Core $^{\mathrm{TM}}$ i5-4200M 2.50GHz CPU and RAM 4.00GB) by using the Microsoft Windows 8 operating system.

4.1. Problem Description. An investor intends to invest his capital in 20 stocks by investing proportion $x_{l}$ in asset $l$, $l=1, \ldots, 20$. After a period of time to investigate the stock market, it is initially locked in the following 20 candidate stocks shown in Table 1. There are 7 stocks selected from Chinese stock market and 13 stocks selected from American stock market. The related parameters are based on the historical data of March 30, 2018. The characteristic of the domestic market rate is low; in general, the maximum rate of stocks should not exceed $10.1 \%$, while the foreign market rate has a large drift. Thus, the appropriate choice of stocks can bring a rich profit.

Each stock has a daily return rate $R_{l}, l=1, \ldots, 20$. Due to the influence of various economic factors and noneconomic factors, the stock return rates constantly change. Figure 1 shows the drift of the SHSP stock daily return rate, from which it is easily observed that the return rate varies around expected value. As a result, consider that the daily return rates $R_{l}, l=1, \ldots, 20$, of the twenty stocks are independent random variables varying in the segments $\left[u_{l}-v_{l}, u_{l}+v_{l}\right]$ around expected values $u_{l}$. Table 1 summarizes these data. For instance, the daily return rate $R_{17}$ of SHSP stock takes its value in $[-2.36,24.49]$, where -2.36 and 24.49 represent the lowest point and the highest point of daily return rate, respectively.

The investor's goal is to determine the optimal investment proportion over the 20 available stocks as return is not less 
TABLE 1: Descriptive statistics for the daily return rates of 20 stocks.

\begin{tabular}{|c|c|c|c|c|}
\hline Decision variable & Stock & Range of $R_{l}(\%)$ & $u_{l}(\%)$ & $v_{l}(\%)$ \\
\hline$x_{1}$ & 002109 & {$[0.40,6.70]$} & 3.55 & 3.15 \\
\hline$x_{2}$ & 002320 & {$[-0.04,9.37]$} & 4.67 & 4.71 \\
\hline$x_{3}$ & 002745 & {$[0.31,7.64]$} & 3.98 & 3.67 \\
\hline$x_{4}$ & 002780 & {$[-0.06,9.97]$} & 4.96 & 5.02 \\
\hline$x_{5}$ & 002858 & {$[0.75,6.15]$} & 3.45 & 2.70 \\
\hline$x_{6}$ & 300263 & {$[-0.61,10.09]$} & 4.74 & 5.35 \\
\hline$x_{7}$ & 300456 & {$[0.03,8.68]$} & 4.36 & 4.33 \\
\hline$x_{8}$ & AGLE & {$[-1.61,16.58]$} & 7.49 & 9.09 \\
\hline$x_{9}$ & CDMO & {$[-1.96,18.76]$} & 8.40 & 10.36 \\
\hline$x_{10}$ & HATR & {$[-1.92,17.79]$} & 8.04 & 9.76 \\
\hline$x_{11}$ & IDN & {$[-0.89,10.88]$} & 5.00 & 5.89 \\
\hline$x_{12}$ & INNT & {$[-1.09,14.01]$} & 6.46 & 7.55 \\
\hline$x_{13}$ & JNP & {$[-2.21,21.35]$} & 9.57 & 11.78 \\
\hline$x_{14}$ & JTPY & {$[-1.08,12.43]$} & 4.68 & 6.69 \\
\hline$x_{15}$ & LENS & {$[-1.05,11.03]$} & 4.99 & 6.04 \\
\hline$x_{16}$ & NES & {$[-1.06,12.58]$} & 5.46 & 6.82 \\
\hline$x_{17}$ & SHSP & {$[-2.36,24.49]$} & 11.07 & 13.43 \\
\hline$x_{18}$ & SRNE & {$[-1.12,15.08]$} & 6.98 & 8.10 \\
\hline$x_{19}$ & STTO & {$[-1.68,16.81]$} & 7.57 & 9.24 \\
\hline$x_{20}$ & $\mathrm{ZN}$ & {$[-2.05,20.20]$} & 9.10 & 11.11 \\
\hline
\end{tabular}

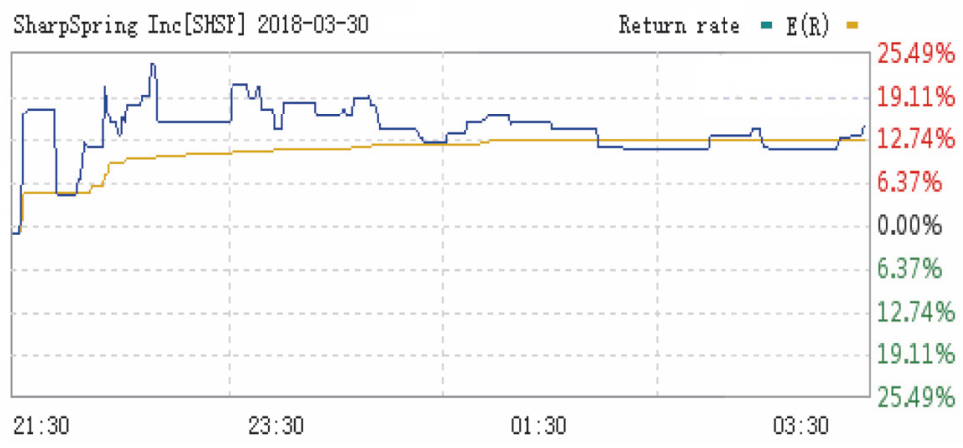

FIGURE 1: The return rate of SHSP.

than a predetermined value. To help the investor make his mind, we can set up the following model:

$$
\begin{array}{ll}
\min & \epsilon \\
\text { s.t. } & \operatorname{Pr}\left\{\sum_{l=1}^{20}\left(R_{l}+1\right) x_{l} \geq \bar{t}\right\} \geq 1-\epsilon, \quad \forall p \in \wp \\
& \sum_{l=1}^{20} x_{l}=1, \quad x_{l} \geq 0, l=1, \ldots, 20,
\end{array}
$$

where $\bar{t}$ is the predetermined value, given in Sections 4.2 and 4.3 , and $\epsilon$ is the risk level. Because the rate $R_{l}$ takes its value in $\left[u_{l}-v_{l}, u_{l}+v_{l}\right]$ around expected values $u_{l}$, it is natural to assume that the natural parameterization of the uncertain return rate is $R_{l}=u_{l}+v_{l} \zeta_{l}, l=1, \ldots, 20$. Under the assumption of the uncertain returns, the chance constraint of model (35) can be rewritten as follows:

$$
\operatorname{Pr}\left\{\sum_{l=1}^{20}\left[\left(u_{l}+1\right)+\zeta_{l} v_{l}\right] x_{l} \geq \bar{t}\right\} \geq 1-\epsilon, \quad \forall p \in \wp .
$$

For the sake of presentation, we introduce symbol $c$ which is the summation of column vectors $c^{0}, c^{1}, \ldots, c^{20}$ with dimension 20; i.e., $c=c^{0}+\sum_{l=1}^{20} \zeta_{l} c^{l}$. Thus, model (35) is equivalent to the following programming:

$$
\begin{array}{ll}
\min & \epsilon \\
\text { s.t. } & \operatorname{Pr}_{\zeta \sim p}\left\{\left[c^{0}+\sum_{l=1}^{20} \zeta_{l} c^{l}\right]^{T} x \geq \bar{t}\right\} \geq 1-\epsilon, \\
& \forall p \in \wp \\
& \sum_{l=1}^{20} x_{l}=1, \quad x_{l} \geq 0, l=1, \ldots, 20,
\end{array}
$$


TABLE 2: Optimal results for different parameters, $\bar{t}=1.02$.

\begin{tabular}{|c|c|c|c|c|c|c|c|c|c|c|}
\hline$\sigma_{l}$ & 0.1 & 0.2 & 0.3 & 0.4 & 0.5 & 0.6 & 0.7 & 0.8 & 0.9 & 1.0 \\
\hline$x_{1}$ & 0.002 & 0.001 & 0.002 & 0.004 & 0.373 & - & - & - & 0.070 & 0.079 \\
\hline$x_{2}$ & 0.001 & 0.001 & 0.001 & 0.009 & 0.028 & 0.002 & 0.011 & 0.127 & 0.002 & 0.002 \\
\hline$x_{3}$ & 0.002 & 0.001 & 0.002 & 0.006 & - & 0.015 & 0.002 & 0.001 & 0.002 & 0.001 \\
\hline$x_{4}$ & - & - & 0.119 & 0.022 & 0.012 & - & - & 0.116 & - & - \\
\hline$x_{5}$ & 0.002 & 0.001 & 0.002 & 0.003 & - & 0.002 & 0.002 & 0.001 & 0.002 & 0.002 \\
\hline$x_{6}$ & 0.309 & 0.035 & 0.015 & 0.033 & 0.009 & - & - & 0.105 & 0.041 & 0.094 \\
\hline$x_{7}$ & 0.001 & 0.001 & 0.002 & 0.007 & - & 0.002 & 0.002 & 0.001 & 0.002 & 0.001 \\
\hline$x_{8}$ & 0.044 & 0.071 & 0.066 & 0.069 & - & - & - & 0.063 & 0.074 & 0.064 \\
\hline$x_{9}$ & 0.040 & 0.142 & 0.058 & 0.061 & 0.058 & 0.166 & 0.168 & 0.055 & 0.066 & 0.056 \\
\hline$x_{10}$ & 0.042 & 0.076 & 0.062 & 0.064 & 0.082 & 0.176 & 0.193 & 0.059 & 0.070 & 0.060 \\
\hline$x_{11}$ & 0.049 & 0.016 & 0.028 & 0.095 & 0.009 & - & - & 0.096 & 0.031 & 0.098 \\
\hline$x_{12}$ & 0.053 & 0.057 & 0.079 & 0.078 & - & - & - & 0.004 & 0.090 & 0.079 \\
\hline$x_{13}$ & 0.036 & 0.080 & 0.051 & 0.053 & 0.083 & 0.146 & 0.148 & 0.049 & 0.058 & 0.050 \\
\hline$x_{14}$ & 0.039 & - & 0.090 & 0.061 & - & - & - & - & 0.017 & - \\
\hline$x_{15}$ & 0.050 & 0.015 & 0.101 & 0.101 & 0.008 & - & - & 0.095 & 0.112 & 0.097 \\
\hline$x_{16}$ & 0.049 & 0.017 & 0.086 & 0.093 & 0.144 & - & - & 0.002 & 0.099 & 0.085 \\
\hline$x_{17}$ & 0.115 & 0.124 & 0.045 & 0.047 & 0.073 & 0.151 & 0.130 & 0.043 & 0.051 & 0.043 \\
\hline$x_{18}$ & 0.051 & 0.070 & 0.074 & 0.080 & - & - & - & 0.071 & 0.083 & 0.072 \\
\hline$x_{19}$ & 0.043 & 0.160 & 0.064 & 0.059 & 0.033 & 0.186 & 0.188 & 0.062 & 0.071 & 0.063 \\
\hline$x_{20}$ & 0.072 & 0.133 & 0.054 & 0.057 & 0.088 & 0.155 & 0.157 & 0.052 & 0.062 & 0.053 \\
\hline
\end{tabular}

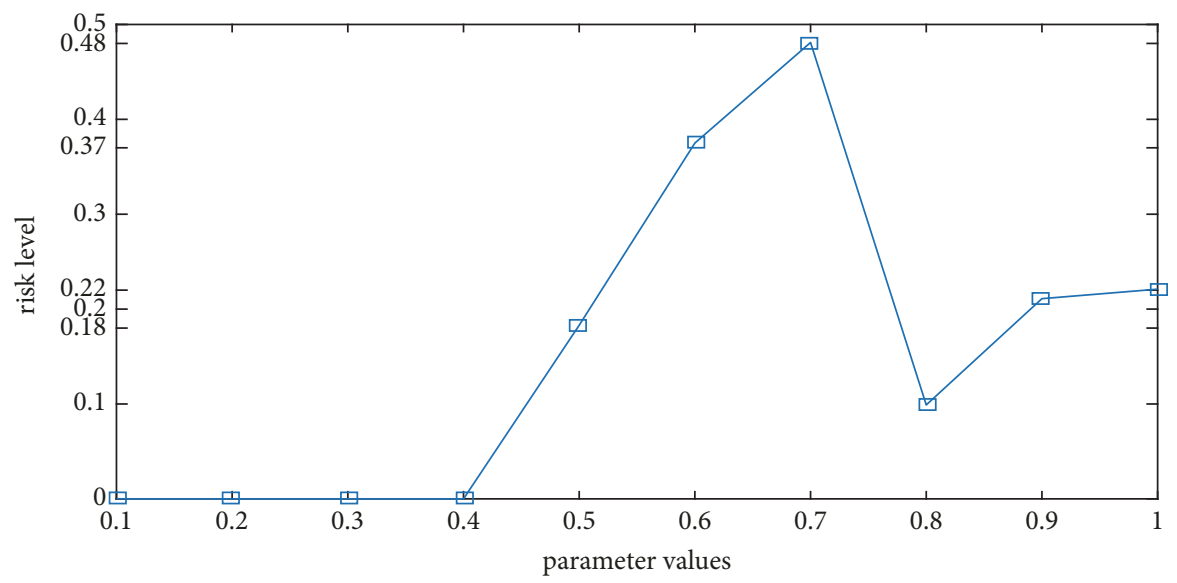

FIGURE 2: Impacts of parameter $\sigma_{l}$ on the risk level, $\bar{t}=1.02$.

where $c^{0}=\left[1+u_{1} ; 1+u_{2} ; 1+u_{3} ; \ldots ; 1+u_{19} ; 1+u_{20}\right]$ and $c^{l}=v_{l} \cdot\left[0_{l-1,1} ; 1 ; 0_{20-l, 1}\right], 1 \leq l \leq 20$.

4.2. Numerical Results with $Z=$ Box+ Generalized Budget. We validate the RC approximation of model (37) with box + generalized budgeted perturbation set (8) for the portfolio selection problem in this subsection. The resulting $\mathrm{RC}$ approximation can be mathematically represented as

$\min \epsilon$

$$
\begin{array}{cl}
\text { s.t. } \quad-\sum_{l=1}^{20}\left|r_{l}\right|-\sqrt{2 \times 20 \ln \left(\frac{1}{\epsilon}\right)} \max _{1 \leq l \leq 20}\left|\sigma_{l} \eta_{l}\right| \\
+\sum_{l=1}^{20}\left(u_{l}+1\right) x_{l} \geq \bar{t}
\end{array}
$$

$$
\begin{aligned}
& r_{l}+\eta_{l}=v_{l} x_{l} \\
& \qquad \sum_{l=1}^{20} x_{l}=1, x_{l} \geq 0, l=1, \ldots, 20 .
\end{aligned}
$$

For the sake of presentation, assume that the parameters $\sigma_{l}$ are equal for each $l$ in model (38). Solving the above model, the optimal investment proportion and minimum risk level are shown in Table 2 and Figure 2 under the parameter $\bar{t}$ $=1.02$.

By Table 2, if the parameter $\sigma_{l}$ takes its value 1 , which corresponds to the usual box + budget uncertainty set, the portfolio selection includes all the stocks except the 4 th and 14 th ones. If the parameter $\sigma_{l}$ takes its value as 
TABLE 3: Optimal results for different return, $\sigma_{l}=0.3$.

\begin{tabular}{|c|c|c|c|c|c|c|c|}
\hline $\bar{t}$ & 1.00 & 1.01 & 1.02 & 1.03 & 1.04 & 1.05 & 1.06 \\
\hline$x_{1}$ & 0.001 & 0.001 & 0.002 & - & - & 0.010 & - \\
\hline$x_{2}$ & 0.001 & 0.001 & 0.001 & - & - & - & - \\
\hline$x_{3}$ & 0.001 & 0.001 & 0.002 & - & - & - & 0.012 \\
\hline$x_{4}$ & 0.163 & 0.263 & 0.119 & - & - & 0.142 & 0.052 \\
\hline$x_{5}$ & 0.002 & 0.001 & 0.002 & - & - & - & - \\
\hline$x_{6}$ & 0.031 & 0.007 & 0.015 & - & - & 0.006 & 0.007 \\
\hline$x_{7}$ & 0.001 & 0.001 & 0.002 & - & - & - & 0.001 \\
\hline$x_{8}$ & 0.077 & 0.075 & 0.066 & 0.154 & 0.148 & 0.079 & 0.098 \\
\hline$x_{9}$ & 0.073 & 0.070 & 0.058 & 0.135 & 0.132 & 0.076 & 0.078 \\
\hline$x_{10}$ & 0.078 & 0.074 & 0.062 & 0.143 & 0.141 & 0.081 & 0.091 \\
\hline$x_{11}$ & 0.040 & - & 0.028 & - & - & 0.038 & 0.047 \\
\hline$x_{12}$ & 0.075 & 0.096 & 0.079 & - & - & 0.100 & 0.099 \\
\hline$x_{13}$ & 0.065 & 0.062 & 0.051 & 0.119 & 0.105 & 0.067 & 0.075 \\
\hline$x_{14}$ & 0.017 & 0.002 & 0.090 & - & - & 0.003 & - \\
\hline$x_{15}$ & 0.036 & 0.019 & 0.101 & - & - & 0.035 & 0.044 \\
\hline$x_{16}$ & 0.050 & 0.034 & 0.086 & - & - & 0.059 & 0.061 \\
\hline$x_{17}$ & 0.056 & 0.054 & 0.045 & 0.163 & 0.092 & 0.059 & 0.066 \\
\hline$x_{18}$ & 0.081 & 0.070 & 0.074 & 0.110 & 0.158 & 0.092 & 0.093 \\
\hline$x_{19}$ & 0.076 & 0.075 & 0.064 & 0.109 & 0.100 & 0.082 & 0.096 \\
\hline$x_{20}$ & 0.075 & 0.094 & 0.054 & 0.067 & 0.124 & 0.069 & 0.080 \\
\hline
\end{tabular}

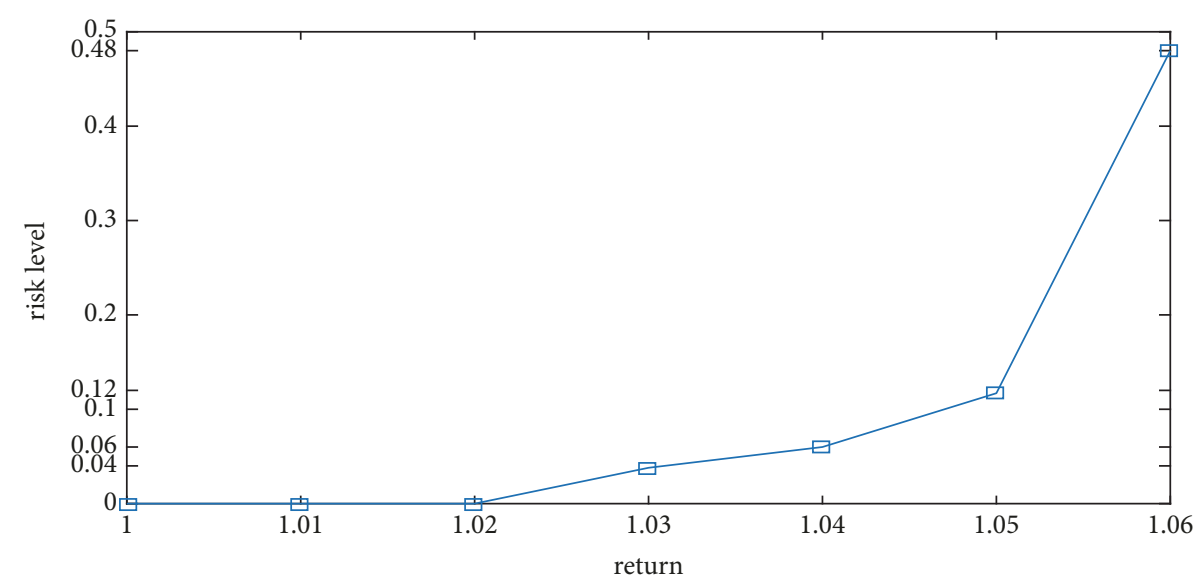

Figure 3: Impacts of return $\bar{t}$ on the risk level, $\sigma_{l}=0.3$.

$0.1,0.2, \ldots, 0.9$, respectively, the recommended investment polices change evidently. For example, when the parameter $\sigma_{l}=0.1$, the investment proportion in the asset 14 is 0.039 , while the investment proportions in asset 14 corresponding to the parameters $0.3,0.4$, and 0.9 are $0.09,0.061$, and 0.017 , respectively. The assets 2 is always invested under different parameters. As a consequence, under different parameters, the recommended investment proportions are different. With different parameters, even if some stocks are always invested, the investment proportions are different.

Under the undesired return $\bar{t}=1.02$, the impacts of parameters on the risk level are plotted in Figure 2, where the horizontal axis corresponds to the parameter and the vertical axis corresponds to the risk level. From Figure 2, it is easily found that the optimal value $\epsilon$ may be larger or smaller under box + generalized budget uncertainty set $\left(\sigma_{l} \neq 1.0\right)$ than the optimal value under box + budget uncertainty set $\left(\sigma_{l}=1.0\right)$. This implies the box + generalized budgeted uncertainty set is less conservative than box + budgeted uncertainty set.

Next we analyze the effects of the $\bar{t}$ on optimal investment proportion and risk level with given parameter $\sigma_{l}=0.3$, $l=1, \ldots, 20$. The computational results are shown in Table 3 and Figure 3. The computational results demonstrate that the recommended investment proportions are different under different return $\bar{t}$. For example, the investor is not recommended to invest the asset 16 under the return 1.03 and 1.04 , while the asset 16 is recommended under the return $1.00,1.01,1.02,1.05$, and 1.06. Moreover, the investment proportions are different even if the asset 16 is always invested. As far as the minimum risk is concerned, it is a 


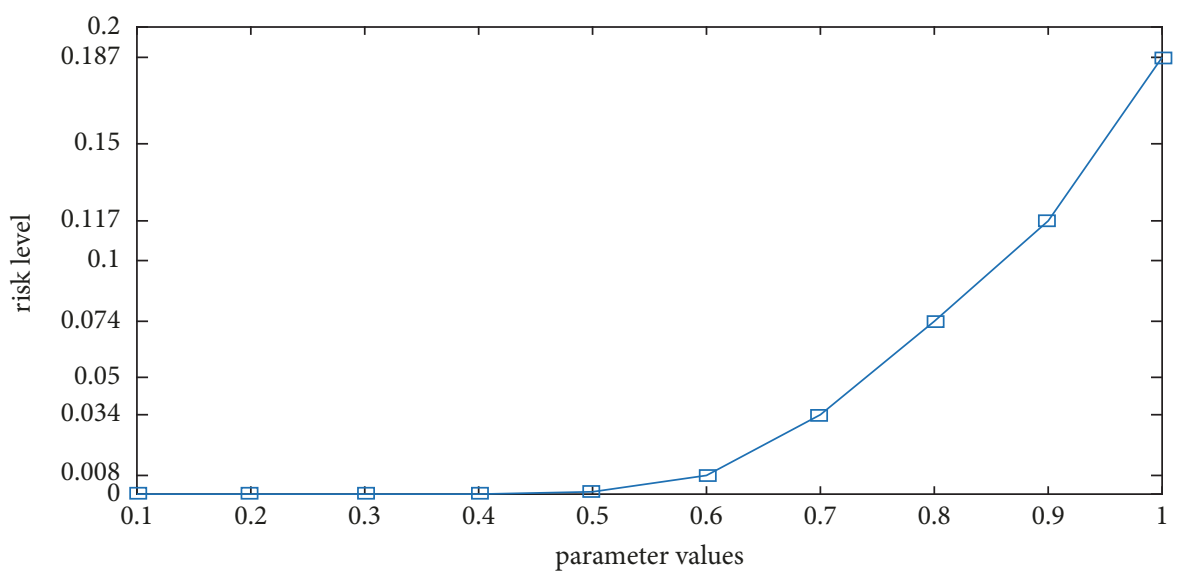

FIgURE 4: Impacts of parameter $\sigma_{l}$ on the risk level, $\bar{t}=1.03$.

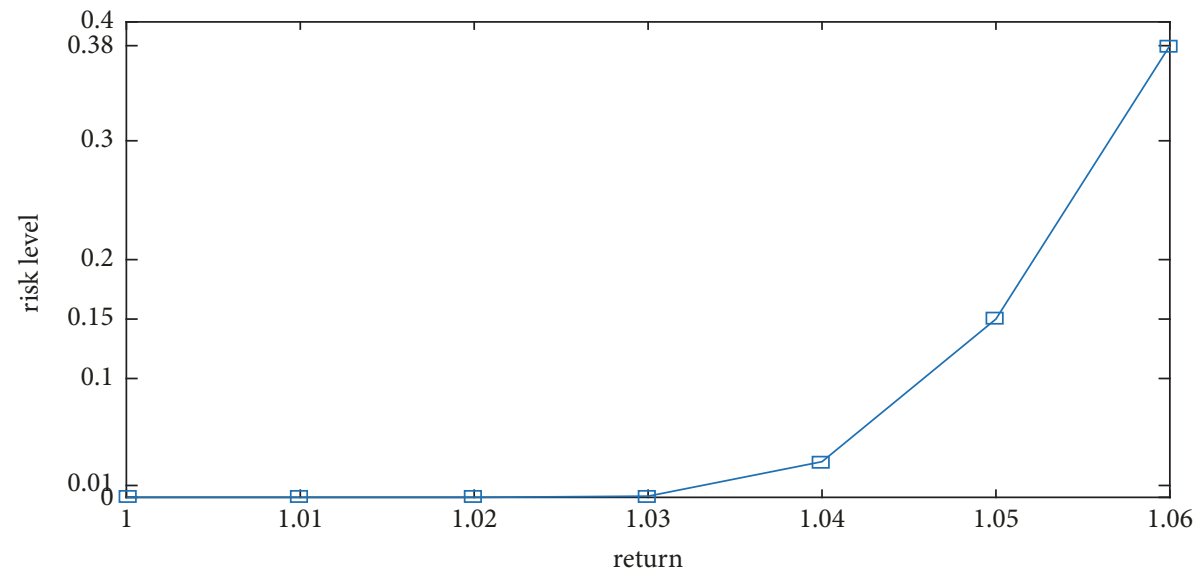

Figure 5: Impacts of return $\bar{t}$ on the risk level, $\sigma_{l}=0.5$.

monotonic trend of the return. That is, with the reduction of the minimum acceptable return, the minimum risk level decreases.

4.3. Numerical Results with $Z=$ Box + Ellipsoid. We discuss the RC approximation of model (37) with box + ellipsoidal perturbation set (25) for the portfolio selection problem in this subsection. The resulting $\mathrm{RC}$ approximation can be mathematically represented as

$$
\begin{array}{ll}
\min & \epsilon \\
\text { s.t. } & -\sum_{l=1}^{20}\left|r_{l}\right|-\sqrt{2 \ln \left(\frac{1}{\epsilon}\right)} \sqrt{\sum_{l=1}^{20} \sigma_{l}^{2} \eta_{l}^{2}}+\sum_{l=1}^{20}\left(u_{l}+1\right) x_{l} \\
\geq \bar{t} \\
r_{l}+\eta_{l}=v_{l} x_{l}, \\
\sum_{l=1}^{20} x_{l}=1, x_{l} \geq 0, l=1, \ldots, 20 .
\end{array}
$$

For simplicity, assume that the parameters $\sigma_{l}$ are equal for each $l$ in model (39). Solving the model, the impacts of parameters on the risk levels are plotted in Figure 4 when setting the undesired return $\bar{t}$ as 1.03 . From Figure 4 , if the parameter $\sigma_{l}$ takes its value 1 , which corresponds to the usual box + ball uncertainty set, the optimal risk level is 0.187 . If the parameter $\sigma_{l}$ takes its value as $0.1,0.2, \ldots, 0.9$, respectively, the associated risk level increases evidently. This shows the different risk levels under different parameters that influence the size of the uncertainty set. At the same time, the optimal risk levels under parameter $\sigma_{l}=0.1,0.2, \ldots, 0.9$, respectively, are less than the risk value of parameter $\sigma_{l}=1, l=1, \ldots, 20$, which shows that box + ellipsoidal uncertainty set is less conservative than box + ball uncertainty set. The impacts of parameters on the risk level are plotted in Figure 5 under the adjustable parameter $\sigma_{l}=0.5$. It is found that the risk level is a monotonically increasing function of the return; i.e., a smaller return leads to a smaller risk level.

The optimal investment proportions for different parameters are provided in Tables 4 and 5. Table 4 reports the different portfolio under parameter $\bar{t}=1.03$ for different $\sigma_{l}$. It is easily checked that the resulting portfolio selections are also different as we change $\bar{t}$. Even if some stocks are always 
TABLE 4: Optimal results for different parameters, $\bar{t}=1.03$.

\begin{tabular}{|c|c|c|c|c|c|c|c|c|c|c|}
\hline$\sigma_{l}$ & 0.1 & 0.2 & 0.3 & 0.4 & 0.5 & 0.6 & 0.7 & 0.8 & 0.9 & 1.0 \\
\hline$x_{1}$ & 0.002 & 0.001 & 0.023 & 0.048 & 0.052 & 0.049 & 0.046 & 0.054 & 0.049 & 0.051 \\
\hline$x_{2}$ & 0.001 & 0.001 & 0.066 & 0.065 & 0.071 & 0.065 & 0.073 & 0.070 & 0.066 & - \\
\hline$x_{3}$ & 0.001 & 0.001 & 0.053 & 0.063 & 0.068 & 0.065 & 0.068 & 0.070 & 0.063 & 0.068 \\
\hline$x_{4}$ & 0.012 & - & 0.078 & 0.067 & 0.034 & 0.068 & - & - & 0.068 & 0.072 \\
\hline$x_{5}$ & 0.002 & 0.001 & 0.019 & 0.053 & 0.055 & 0.052 & 0.044 & 0.060 & 0.051 & 0.057 \\
\hline$x_{6}$ & 0.010 & - & 0.056 & 0.053 & 0.057 & 0.053 & 0.058 & 0.055 & 0.053 & 0.056 \\
\hline$x_{7}$ & 0.001 & 0.001 & 0.058 & 0.063 & 0.068 & 0.064 & 0.068 & 0.070 & 0.063 & 0.067 \\
\hline$x_{8}$ & 0.008 & 0.058 & 0.050 & 0.047 & 0.051 & 0.047 & 0.052 & 0.050 & 0.047 & 0.050 \\
\hline$x_{9}$ & 0.007 & 0.105 & 0.047 & 0.044 & 0.046 & 0.043 & 0.048 & 0.046 & 0.044 & 0.047 \\
\hline$x_{10}$ & 0.007 & 0.165 & 0.049 & 0.046 & 0.049 & 0.046 & 0.050 & 0.049 & 0.046 & 0.049 \\
\hline$x_{11}$ & 0.009 & 0.005 & 0.059 & 0.050 & 0.026 & 0.050 & 0.054 & 0.053 & 0.050 & 0.054 \\
\hline$x_{12}$ & 0.009 & 0.037 & 0.056 & 0.053 & 0.057 & 0.053 & 0.058 & 0.056 & 0.053 & 0.056 \\
\hline$x_{13}$ & 0.007 & 0.136 & 0.044 & 0.041 & 0.042 & 0.041 & 0.045 & 0.044 & 0.041 & 0.044 \\
\hline$x_{14}$ & 0.006 & - & 0.033 & 0.033 & 0.035 & 0.032 & 0.038 & 0.034 & 0.033 & 0.035 \\
\hline$x_{15}$ & 0.009 & 0.005 & 0.057 & 0.047 & 0.051 & 0.047 & 0.051 & 0.050 & 0.047 & 0.051 \\
\hline$x_{16}$ & 0.008 & 0.010 & 0.053 & 0.046 & 0.049 & 0.046 & 0.050 & 0.048 & 0.046 & 0.049 \\
\hline$x_{17}$ & 0.102 & 0.135 & 0.043 & 0.039 & 0.040 & 0.038 & 0.042 & 0.041 & 0.039 & 0.042 \\
\hline$x_{18}$ & 0.009 & 0.052 & 0.060 & 0.053 & 0.057 & 0.052 & 0.057 & 0.056 & 0.053 & 0.056 \\
\hline$x_{19}$ & 0.008 & 0.058 & 0.052 & 0.046 & 0.050 & 0.046 & 0.051 & 0.049 & 0.047 & 0.050 \\
\hline$x_{20}$ & 0.782 & 0.229 & 0.046 & 0.043 & 0.044 & 0.043 & 0.047 & 0.046 & 0.043 & 0.046 \\
\hline
\end{tabular}

TABLE 5: Optimal results for different return, $\sigma_{l}=0.5$.

\begin{tabular}{lccccccc}
\hline $\bar{t}$ & 1.00 & 1.01 & 1.02 & 1.03 & 1.04 & - & 1.05 \\
\hline$x_{1}$ & 0.002 & 0.002 & 0.002 & 0.052 & - & - & - \\
$x_{2}$ & 0.147 & 0.096 & 0.081 & 0.071 & 0.045 & - \\
$x_{3}$ & 0.002 & 0.002 & 0.096 & 0.068 & - & - \\
$x_{4}$ & - & 0.097 & 0.079 & 0.034 & - & - & - \\
$x_{5}$ & 0.002 & 0.002 & 0.002 & 0.055 & - & - \\
$x_{6}$ & 0.098 & 0.089 & 0.065 & 0.057 & 0.048 & - \\
$x_{7}$ & 0.002 & 0.002 & 0.084 & 0.068 & 0.018 & - \\
$x_{8}$ & 0.055 & 0.050 & 0.042 & 0.051 & 0.078 & 0.104 \\
$x_{9}$ & 0.047 & 0.048 & 0.041 & 0.046 & 0.075 & 0.110 \\
$x_{10}$ & 0.049 & 0.047 & 0.044 & 0.049 & 0.078 & 0.110 \\
$x_{11}$ & 0.085 & 0.079 & 0.057 & 0.026 & 0.054 & - \\
$x_{12}$ & 0.066 & 0.060 & 0.050 & 0.057 & 0.080 & 0.099 \\
$x_{13}$ & 0.046 & 0.041 & 0.037 & 0.042 & 0.074 & 0.123 \\
$x_{14}$ & 0.054 & 0.054 & 0.047 & 0.035 & 0.029 & 0.118 \\
$x_{15}$ & 0.085 & 0.070 & 0.055 & 0.051 & 0.051 & - \\
$x_{16}$ & 0.067 & 0.064 & 0.050 & 0.049 & 0.058 & - \\
$x_{17}$ & 0.037 & 0.038 & 0.033 & 0.040 & 0.073 & - \\
$x_{18}$ & 0.062 & 0.064 & 0.052 & 0.057 & 0.044 \\
$x_{19}$ & 0.047 & 0.051 & 0.041 & 0.050 & 0.084 & - \\
$x_{20}$ & 0.046 & 0.044 & 0.040 & 0.044 & 0.074 & - \\
\hline
\end{tabular}

invested, the investment proportions are also different. For example, when the parameter $\sigma_{l}$ is 0.1 , the investment proportion in asset 20 is 0.782 , while the investment proportion in asset 20 is 0.229 under the parameter $\sigma_{l}=0.2$. Furthermore, Table 5 displays the influence of $\bar{t}$ on optimal investment proportions with given parameter $\sigma_{l}=0.5, l=1, \ldots, 20$, from which it is observed that the resulting portfolio selections are also different as we change $\bar{t}$. Although the asset 20 is always invested, the investment proportions are different.

The numerical experiments demonstrate that ambiguous P-model can provide diversification resulting portfolios to assets. When the parameter $\sigma_{l}$ and undesired return $\bar{t}$ change, 
TABLE 6: Model comparison results under $\bar{t}=1.02$ and $\sigma_{l}=1$.

\begin{tabular}{lccccccc}
\hline$x$ & $\begin{array}{c}\text { Nominal } \\
\text { solutions }\end{array}$ & $\begin{array}{c}\text { Ellipsoidal } \\
\text { solutions }\end{array}$ & $\begin{array}{c}\text { Generalized } \\
\text { budgeted } \\
\text { solutions }\end{array}$ & $x$ & $\begin{array}{c}\text { Nominal } \\
\text { solutions }\end{array}$ & $\begin{array}{c}\text { Ellipsoidal } \\
\text { solutions }\end{array}$ & $\begin{array}{c}\text { Generalized } \\
\text { budgeted } \\
\text { solutions }\end{array}$ \\
\hline$x_{1}$ & 0.08510695 & 0.08783705 & 0.07935254 & $x_{11}$ & 0.04711347 & 0.047479 & 0.09826442 \\
$x_{2}$ & 0.06557282 & 0.06475769 & 0.00158102 & $x_{12}$ & 0.04262804 & 0.04291164 & 0.07936592 \\
$x_{3}$ & 0.0800917 & 0.077931940 & 0.00145014 & $x_{13}$ & 0.02972075 & 0.0298708 & 0.04955437 \\
$x_{4}$ & 0.06399392 & 0.06454947 & - & $x_{14}$ & 0.03262398 & 0.03289855 \\
$x_{5}$ & 0.1083665 & 0.1056714 & 0.00161524 & $x_{15}$ & 0.04465311 & 0.04500051 & 0.09664661 \\
$x_{6}$ & 0.0521551 & 0.05258966 & 0.09423644 & $x_{16}$ & 0.04052862 & 0.04081349 & 0.08492115 \\
$x_{7}$ & 0.06857892 & 0.06743896 & 0.00147484 & $x_{17}$ & 0.02739742 & 0.0275358 & 0.04346616 \\
$x_{8}$ & 0.0361992 & 0.03638232 & 0.06421459 & $x_{18}$ & 0.04135364 & 0.04152863 & 0.07198850 \\
$x_{9}$ & 0.03248739 & 0.03255628 & 0.05634589 & $x_{19}$ & 0.03554395 & 0.03586811 & 0.06316887 \\
$x_{10}$ & 0.03454552 & 0.03473147 & 0.05981051 & $x_{20}$ & 0.03133897 & 0.03164698 & 0.05254279 \\
\hline
\end{tabular}

respectively, the risk level and invested stocks vary accordingly. Even if the invested stocks are the same, the invested proportions to them are usually different. As a result, the results illustrate that $\mathrm{RC}$ approximations of the ambiguous P-model constitute a new method for modeling portfolio selection problem with the uncertain return rates, which can provide diversification investments for decision makers.

4.4. Comparison Study with Nominal Stochastic Model. In order to evaluate the advantage of the proposed model, comparison study with nominal stochastic model is conducted. Under $(P 1)$ and $(P 2)$, we consider that $\zeta_{l}, l=1, \ldots, L$, are independent Gaussian random variables. The expectation and variance of the random variable $\zeta_{l}$ are $\mu_{l}$ and $\sigma_{l}$, which was proved in [16]. Without loss of generality, we assume the $\sigma_{l}=1$ as well as $\mu_{l}=0$ shown in (P2). Therefore, the uncertain return rate $R_{l}=u_{l}+v_{l} \zeta_{l}$ obeys a Gaussian distribution with expectation $u_{l}$ and variance $v_{l}, l=1, \ldots, L$. Based on the above analysis, the nominal stochastic model can be written as

$$
\begin{array}{ll}
\min & \epsilon \\
\text { s.t. } & \operatorname{Pr}\left\{\sum_{l=1}^{20}\left(R_{l}+1\right) x_{l} \geq \bar{t}\right\} \geq 1-\epsilon, \\
& \sum_{l=1}^{20} x_{l}=1, \quad x_{l} \geq 0, l=1, \ldots, 20 .
\end{array}
$$

By standardization of Gaussian distribution, model (40) is equivalent to the following form:

$$
\begin{array}{ll}
\min & \epsilon \\
\text { s.t. } & \Phi\left(\frac{\bar{t}-\sum_{l=1}^{20}\left(u_{l}+1\right) x_{l}}{\sqrt{\sum_{l=1}^{20} x_{l}^{2} \sigma_{l}^{2}}}\right) \leq \epsilon, \\
& \sum_{l=1}^{20} x_{l}=1, \quad x_{l} \geq 0, l=1, \ldots, 20 .
\end{array}
$$

Since the standard normal distribution function $\Phi(\cdot)$ in model (41) is monotonic increasing, we only solve the following equivalent model:

$$
\begin{array}{ll}
\min & \frac{\bar{t}-\sum_{l=1}^{20}\left(u_{l}+1\right) x_{l}}{\sqrt{\sum_{l=1}^{20} x_{l}^{2} \sigma_{l}^{2}}} \\
\text { s.t. } & \sum_{l=1}^{20} x_{l}=1, \quad x_{l} \geq 0, \quad l=1, \ldots, 20 .
\end{array}
$$

After that, we can obtain $\epsilon$ according to Gaussian distribution table.

As far as optimal solutions are concerned, the comparison results with $\bar{t}=1.02$ and $\sigma_{l}=1$ are shown in Table 6 . It is evident that stochastic solutions are different from robust solutions under the box + ellipsoidal and box + generalized budgeted uncertainty sets. From Table 6, we can observe that the invested assets and investment proportions are both different between nominal stochastic model and robust model under box + generalized budgeted uncertainty set. For example, the assets 4 and 14 are not invested for robust model under the box + generalized budgeted uncertainty set, while the assets 4 and 14 are invested for nominal stochastic model. Furthermore, we can observe that the investment proportions are different even if the assets are always invested between stochastic and robust models. For example, the investment proportion in asset 2 is 0.06557282 for nominal stochastic model, while the investment proportions in asset 2 are 0.06475769 and 0.00158102 for robust model under the box + ellipsoidal and box + generalized budgeted uncertainty sets, respectively. Therefore, uncertain distribution can impact the optimal strategies to a certain extent, which effectively illustrates that uncertainty cannot be ignored.

With respect to optimal value, we further investigate the comparison results on risk level between nominal stochastic model and robust model. From Figure 6, it can be found that risk level of nominal stochastic model is evidently less than those of the robust models under the box + ellipsoidal and box + generalized budgeted uncertainty sets. This phenomenon is perfectly consistent with the theoretical 


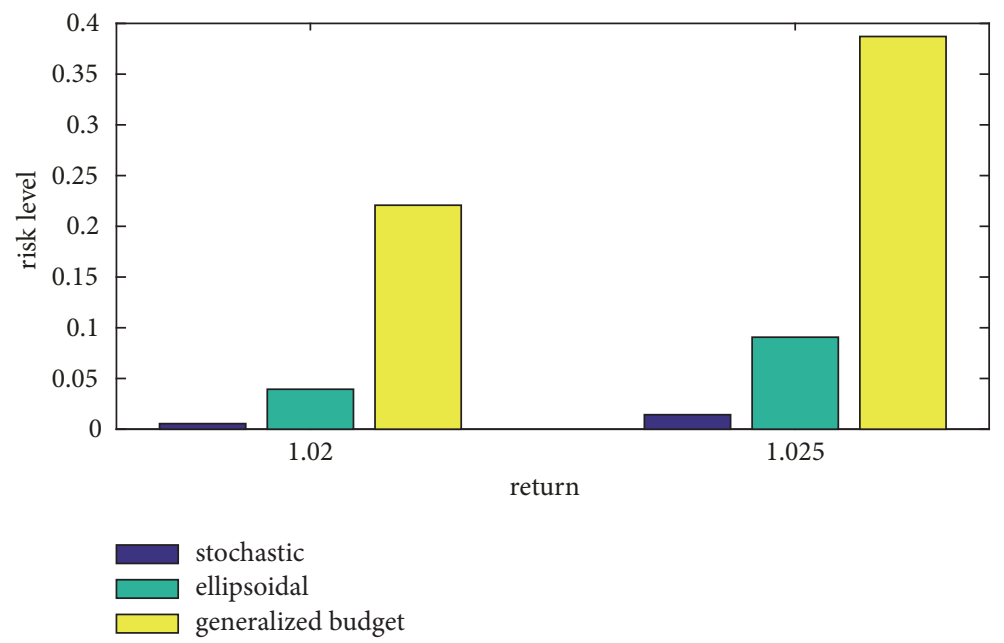

FIGURE 6: Robust risk level versus stochastic risk level, $\sigma_{l}=1$.

facts. This is principally because of the existence of uncertain distribution. Although the robust models incur higher risk level, they can resist the uncertainty better than nominal stochastic model. In real-world problem, the exact distribution information is often unavailable, which drives us to study the robust optimization model.

\section{Conclusions}

In the present paper, a kind of ambiguous P-model was addressed to cope with the uncertainty. We assumed that the uncertain parameters possessed two special properties and varied in the box + generalized budgeted and box + ellipsoidal uncertainty sets. Due to computational challenge of the ambiguous P-model, we built the safe tractable approximations of the model under two refined uncertainty sets by robust optimization approach. The original model thus was transformed into the nonlinear programming, which can be solved by the common software such as Lingo. To verify the power of ambiguous P-model, we considered a portfolio selection problem with uncertain return rates. The numerical experiments showed that our model can effectively be applied to portfolio selection problem under uncertain return rates varying in the refined uncertainty sets. In addition, our refined uncertainty sets were less conservative than the unrefined uncertainty sets when parameter $\sigma_{l}$ took some certain values, and provided more diversified investment decision for investors. By comparison study with nominal stochastic model, it was proved that uncertain information had a great influence on the optimal decisions. That is to say, the uncertain information cannot be ignored and our ambiguous P-model can resist the uncertainty better than the nominal stochastic model.

Several extensions of this work are possible. For example, the robust chance constraint model should further consider the safe convex approximation under other conditions such as known variance, asymmetry, and unimodality, as well as known range, mean, and variance. In addition to portfolio selection problem, we can apply further ambiguous P-model to study other domains: emergency supplies prepositioning and allocation problem [38], urban sustainable development and management, water resource management, supply chain network design problem [39], and so on.

\section{Data Availability}

The data used to support the findings of this study are available from the corresponding author upon request.

\section{Conflicts of Interest}

The authors declare that they have no conflicts of interest.

\section{Acknowledgments}

This work is partially supported by the National Natural Science Foundation of China (no. 61773150) and the Youth Natural Science Foundation of Hebei Province (no. A2016204057).

\section{References}

[1] A. L. Soyster, "Convex programming with set-inclusive constraints and applications to inexact linear programming," Operations Research, vol. 21, no. 5, pp. 1154-1157, 1973.

[2] A. Ben-Tal and A. Nemirovski, "Robust convex optimization," Mathematics of Operations Research, vol. 23, no. 4, pp. 769-805, 1998.

[3] A. Ben-Tal and A. Nemirovski, "Robust solutions of uncertain linear programs," Operations Research Letters, vol. 25, no. 1, pp. 1-13, 1999.

[4] D. Bertsimas, D. Pachamanova, and M. Sim, "Robust linear optimization under general norms," Operations Research Letters, vol. 32, no. 6, pp. 510-516, 2003.

[5] D. Bertsimas and M. Sim, "Tractable approximations to robust conic optimization problems," Mathematical Programming, vol. 107, no. 1, pp. 5-36, 2006. 
[6] V. Gabrel, C. Murat, and A. Thiele, "Recent advances in robust optimization: an overview," European Journal of Operational Research, vol. 235, no. 3, pp. 471-483, 2014.

[7] X. Bai and Y. K. Liu, "Robust optimization of supply chain network design in fuzzy decision system," Journal of Intelligent Manufacturing, vol. 27, no. 6, pp. 1131-1149, 2016.

[8] A. Jabbarzadeh, M. Haughton, and A. Khosrojerdi, "Closedloop supply chain network design under disruption risks: a robust approach with real world application," Computers \& Industrial Engineering, vol. 116, pp. 178-191, 2018.

[9] H. Omrani, "Common weights data envelopment analysis with uncertain data: A robust optimization approach," Computers \& Industrial Engineering, vol. 66, no. 4, pp. 1163-1170, 2013.

[10] A. H. Shokouhi, A. Hatami-Marbini, M. Tavana, and S. Saati, "A robust optimization approach for imprecise data envelopment analysis," Computers \& Industrial Engineering, vol. 59, no. 3, pp. 387-397, 2010.

[11] M. E. Bruni, L. Di Puglia Pugliese, P. Beraldi, and F. Guerriero, "An adjustable robust optimization model for the resourceconstrained project scheduling problem with uncertain activity durations," OMEGA - The International Journal of Management Science, vol. 71, pp. 66-84, 2017.

[12] S. Neyshabouri and B. P. Berg, "Two-stage robust optimization approach to elective surgery and downstream capacity planning," European Journal of Operational Research, vol. 260, no. 1, pp. 21-40, 2017.

[13] Q. W. Liu, Z. Z. Guo, and Y. J. Chen, "Distributionally robust multi-product newsvendor problem under carbon policies," Journal of Uncertain Systems, vol. 12, no. 1, pp. 56-67, 2018.

[14] Y. Liu, H. Lei, D. Zhang, and Z. Wu, "Robust optimization for relief logistics planning under uncertainties in demand and transportation time," Applied Mathematical Modelling: Simulation and Computation for Engineering and Environmental Systems, vol. 55, pp. 262-280, 2018.

[15] Z. Zhang and H. Jiang, "A robust counterpart approach to the biobjective emergency medical service design problem," Applied Mathematical Modelling, vol. 38, no. 3, pp. 1033-1040, 2014.

[16] A. Ben-Tal, L. El Ghaoui, and A. Nemirovski, Robust optimization, Princeton Series in Applied Mathematics, Princeton University Press, Princeton, NJ, USA, 2009.

[17] Z. Li and Z. Li, "Optimal robust optimization approximation for chance constrained optimization problem," Computers \& Chemical Engineering, vol. 74, pp. 89-99, 2015.

[18] A. Nemirovski, "On safe tractable approximations of chance constraints," European Journal of Operational Research, vol. 219, no. 3, pp. 707-718, 2012.

[19] R. Duzgun and A. Thiele, "Robust binary optimization using a safe tractable approximation," Operations Research Letters, vol. 43, no. 4, pp. 445-449, 2015.

[20] W. Yang and H. Xu, "Distributionally robust chance constraints for non-linear uncertainties," Mathematical Programming, vol. 155, no. 1-2, Ser. A, pp. 231-265, 2016.

[21] K. Postek, A. Ben-Tal, D. den Hertog, and B. Melenberg, "Robust optimization with ambiguous stochastic constraints under mean and dispersion information," Operations Research, vol. 66 , no. 3, pp. 814-833, 2018.

[22] R. Qiu, J. Shang, and X. Huang, "Robust inventory decision under distribution uncertainty: A CVaR-based optimization approach," International Journal of Production Economics, vol. 153, pp. 13-23, 2014.
[23] S. Zymler, D. Kuhn, and B. Rustem, "Distributionally robust joint chance constraints with second-order moment information," Mathematical Programming, vol. 137, no. 1-2, pp. 167-198, 2013.

[24] X. J. Bai and Y. K. Liu, "CVAR reduced fuzzy variables and their second order moments," Iranian Journal of Fuzzy Systems, vol. 12, no. 5, pp. 45-75, 2015.

[25] N. Q. Liu, Y. J. Chen, and Y. K. Liu, "Optimization portfolio selection problems under credibilistic CVaR criterion," Journal of Intelligent \& Fuzzy Systems: Applications in Engineering and Technology, vol. 34, no. 1, pp. 335-347, 2018.

[26] R. Bruni, F. Cesarone, A. Scozzari, and F. Tardella, "On exact and approximate stochastic dominance strategies for portfolio selection," European Journal of Operational Research, vol. 259, no. 1, pp. 322-329, 2017.

[27] A.-F. Ling and C.-X. Xu, "Robust portfolio selection involving options under a "marginal + joint" ellipsoidal uncertainty set," Journal of Computational and Applied Mathematics, vol. 236, no. 14, pp. 3373-3393, 2012.

[28] N. Gulpinar and E. Canakoglu, "Robust portfolio selection problem under temperature uncertainty," European Journal of Operational Research, vol. 256, no. 2, pp. 500-523, 2017.

[29] C. Gregory, K. Darby-Dowman, and G. Mitra, "Robust optimization and portfolio selection: the cost of robustness," European Journal of Operational Research, vol. 212, no. 2, pp. 417-428, 2011.

[30] A. Charnes and W. W. Cooper, "Chance-constrained programming," Management Science, vol. 6, no. 1, pp. 73-79, 1959.

[31] A. Charnes and W. W. Cooper, "Deterministic equivalents for optimizing and satisficing under chance constraints," Operations Research, vol. 11, pp. 18-39, 1963.

[32] A. Charnes and M. J. Kirby, "Some special P-models in chanceconstrained programming," Management Science, vol. 14, pp. 183-195, 1967.

[33] P. Walley, Statistical Reasoning with Imprecise Probabilities, vol. 42 of Monographs on Statistics and Applied Probability, Chapman and Hall, Ltd, London, UK, 1st edition, 1991.

[34] P. Walley, R. Pelessoni, and P. Vicig, "Direct algorithms for checking consistency and making inferences from conditional probability assessments," Journal of Statistical Planning and Inference, vol. 126, no. 1, pp. 119-151, 2004.

[35] F. G. Cozman and P. Walley, "Graphoid properties of epistemic irrelevance and independence," Annals of Mathematics and Artificial Intelligence, vol. 45, no. 1-2, pp. 173-195, 2005.

[36] J. Atlason, M. A. Epelman, and S. G. Henderson, "Optimizing call center staffing using simulation and analytic center cuttingplane methods," Management Science, vol. 54, no. 2, pp. 295309, 2008.

[37] A. Nemirovski and A. Shapiro, "Convex approximations of chance constrained programs," SIAM Journal on Optimization, vol. 17, no. 4, pp. 969-996, 2006.

[38] X. Bai, “Two-Stage multiobjective optimization for emergency supplies allocation problem under integrated uncertainty," Mathematical Problems in Engineering, vol. 2016, Article ID 2823835, pp. 1-13, 2016.

[39] Y. K. Liu, X. J. Bai, and K. Yang, Parametric Credibilistic Optimization Methods, Science Press, Beijing, China, 2017. 


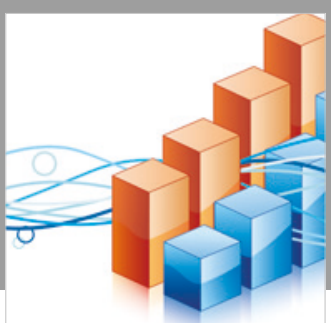

Advances in

Operations Research

\section{-n-m}
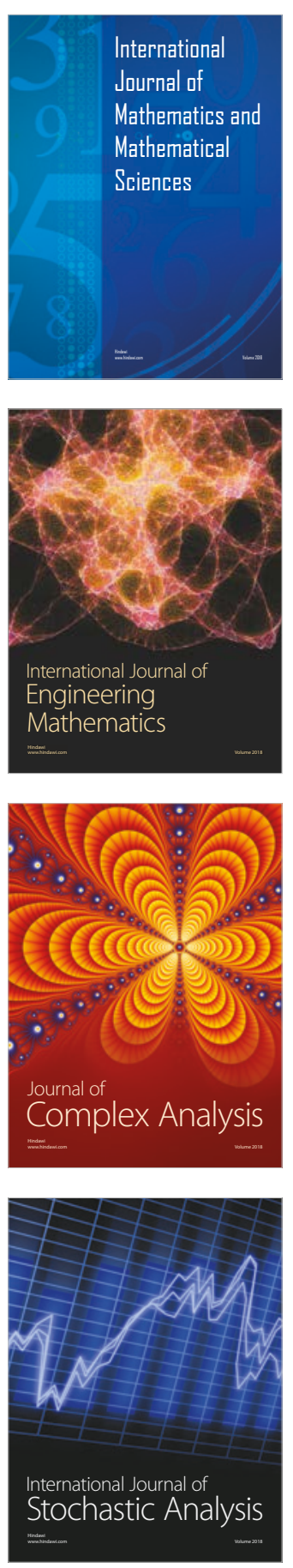
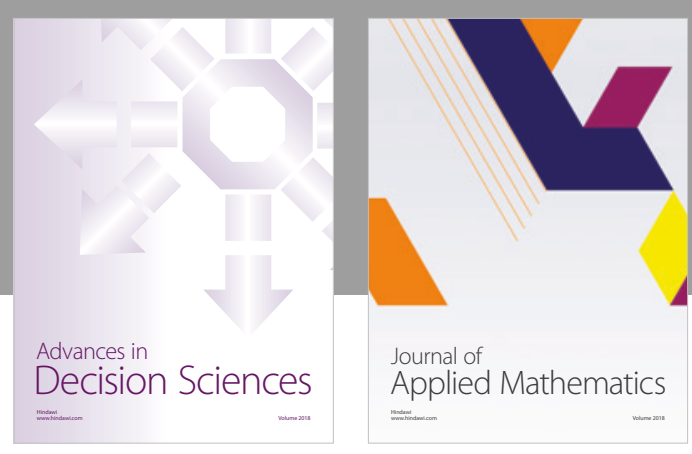

Journal of

Applied Mathematics
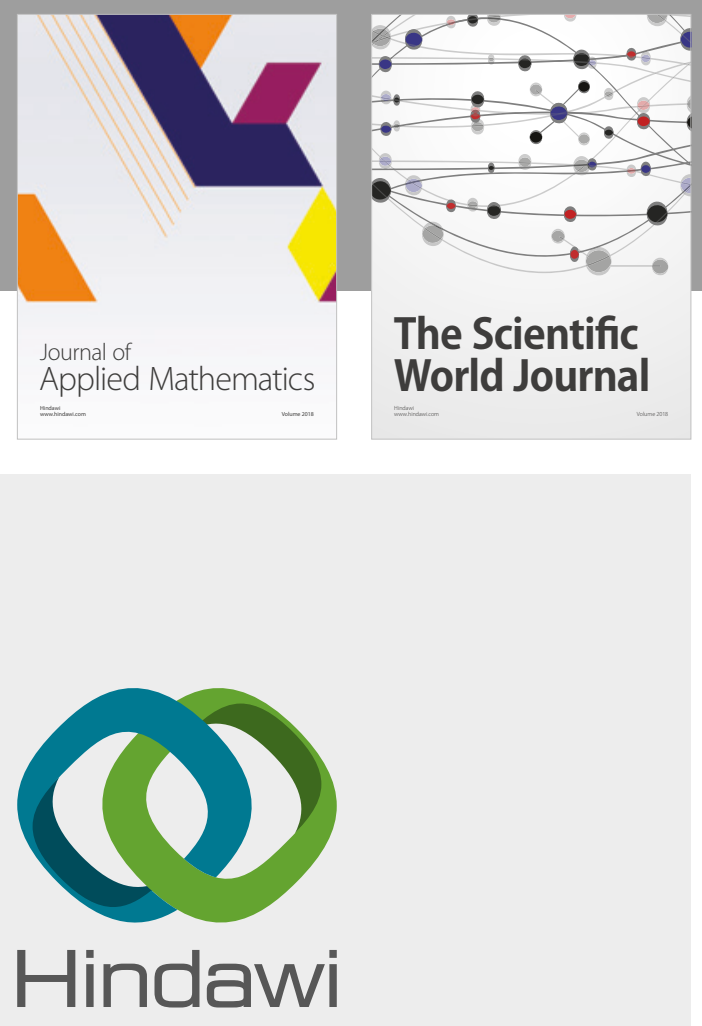

Submit your manuscripts at

www.hindawi.com

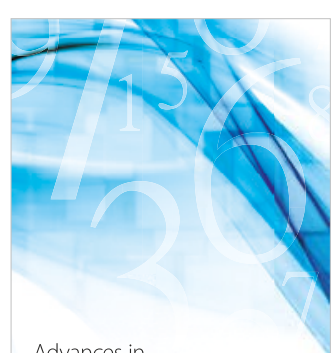

Advances in
Numerical Analysis
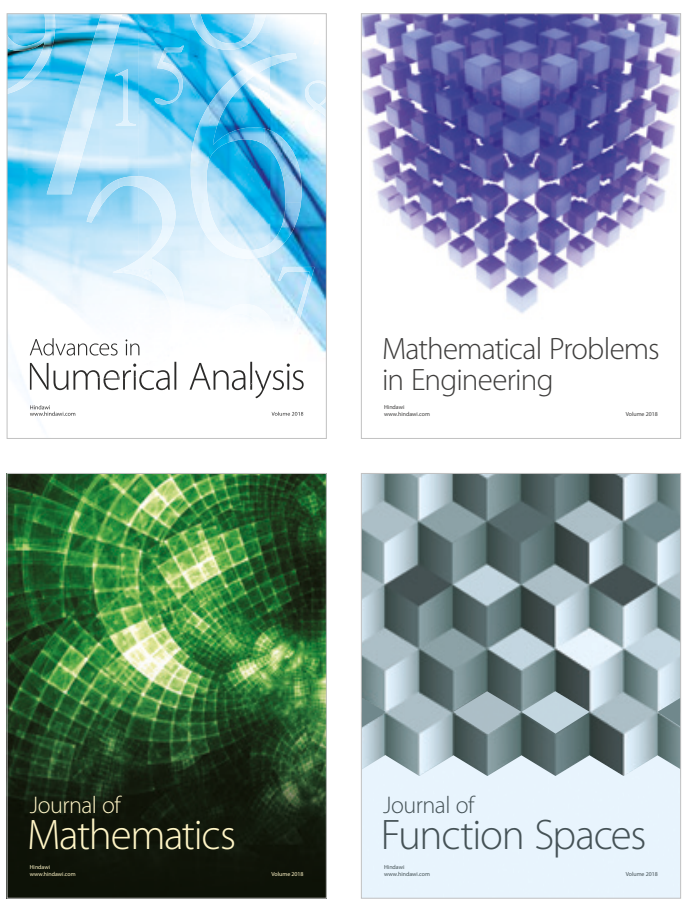

Mathematical Problems in Engineering

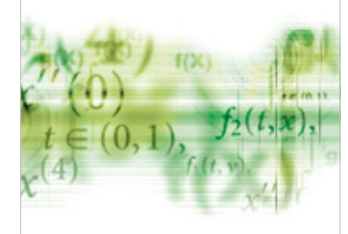

International Journal of

Differential Equations

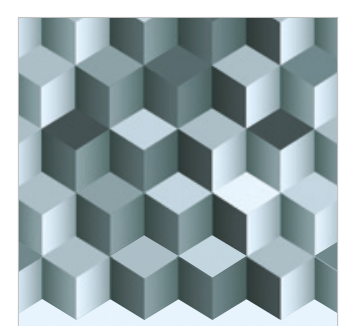

Journal of

Function Spaces

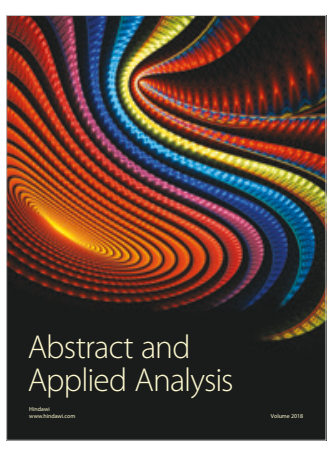

The Scientific

World Journal

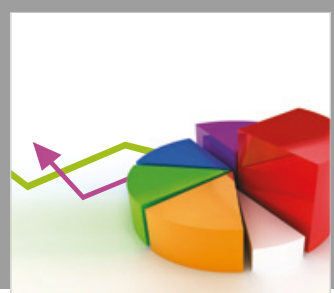

Journal of

Probability and Statistics
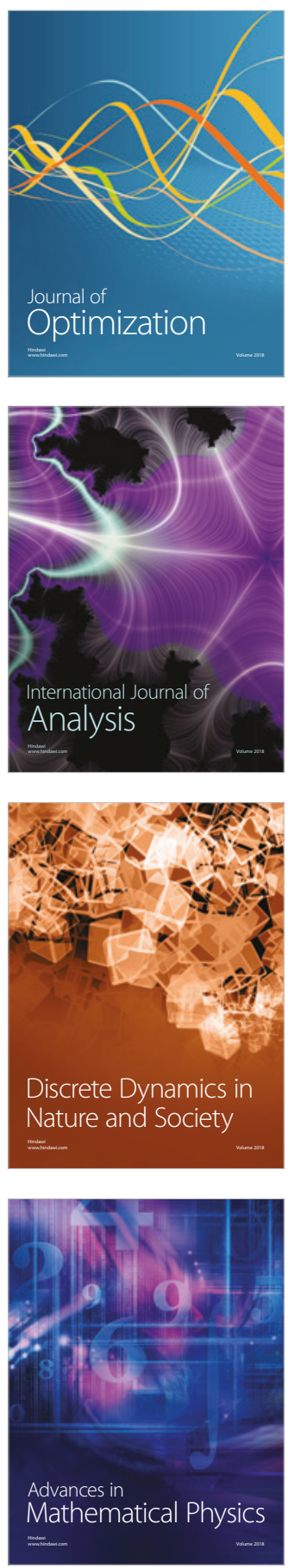